\title{
Dysregulation of Diverse Ion Transport Pathways Controlling Cell Volume Homoestasis Contribute to Neuroglial Cell Injury Following Ischemic Stroke
}

\author{
Dandan Sun • Kristopher T. Kahle
}

Received: 5 January 2014 / Accepted: 5 January 2014 /Published online: 27 January 2014

(C) The Author(s) 2014. This article is published with open access at Springerlink.com

This special featured issue of Translational Stroke Research gives readers a timely update on the emerging roles of glutamate receptor-independent ion channels and transporters in ischemic brain injury. This issue has collected contributions from the leading research laboratories with expertise on the most important ion transport targets. For example, Khanna et al. [1] provide an overview on the significance of dysregulated ion homeostasis in the neurogliovascular unit in the pathogenesis of ischemic cerebral edema, focusing on the role of the sulfonylurea receptor 1-transient receptor potential melastatin 4 (SUR1-TRPM4) channel complex which is upregulated in the context of hypoxia and promotes capillary fragmentation and oncotic cell death in the vascular endothelium, thereby increasing vascular permeability and vasogenic edema. Song et al. write an elegant review on the distinct changes of cell volume homeostasis in necrotic, apoptotic, and hybrid cell death that accompany ischemic stroke, along with the $N$-methyl-D-aspartate-independent ion transporters and channels that mediate apoptotic volume decrease or cytotoxic cell swelling [2]. Norenberg et al. [3] report novel mechanisms underlying astrocyte swelling and brain edema mediated by SUR1-TRPM4 activation in acute liver failure, extending original findings by the Simard group in ischemic disease to other types of brain swelling.

D. Sun $(\bowtie)$

Department of Neurology, University of Pittsburgh School of

Medicine, Pittsburgh, PA 15217, USA

e-mail: sund@upmc.edu

K. T. Kahle

Department of Neurosurgery, Massachusetts General Hospital and Harvard Medical, Boston, MA 02115, USA

K. T. Kahle

Manton Center for Orphan Disease Research, Children's Hospital Boston, Boston, MA 02115, USA
The second half of the issue include comprehensive discussions of voltage-gated $\mathrm{K}^{+}$channels [4] and the acidsensing ion channel 1a ASIC1a [5, 6] in ischemic neurotoxicity, and voltage-gated $\mathrm{H}^{+}$channel $\mathrm{Hvl}$ in microglial activation [7], following stroke. Uria-Avellanal et al. [8] present compelling clinical and experimental research data on overstimulation of $\mathrm{Na}^{+} / \mathrm{H}^{+}$exchangers and alkaline $\mathrm{pHi}$ in perinatal brain injury. Pignataro et al. discuss a strategy of targeting increased $\mathrm{Ca}^{2+}$ efflux mediated by $\mathrm{Na}^{+} / \mathrm{Ca}^{2+}$ exchanger in stroke intervention [9]. Chen et al. review the significance of ion channels in regulation of neuronal progenitor function in ischemic brains, which holds the potential for tissue regeneration - an emerging area in the field of neuroprotection [10].

In summary, these timely papers illustrate exciting new findings in the area of glutamate receptor-independent mechanisms of ischemic ionic injury in the nervous system, and reveal the critical role of dysregulated ion transport, mediated by diverse ion transport proteins, in the neurogliovascular unit. These reports show that ischemia-induced ion channel/ transporter dysfunction affects multiple critical cell processes, including cell volume regulation [11], intracellular ionic homeostasis [12], neuroinflammation [13, 14], and neuroregeneration/tissue repair $[15,16]$. An increased understanding of the role these transport proteins play in normal biology, along with further knowledge of how these proteins are regulated by post-translational modification and signal transduction, might reveal novel methods by which these transport systems may be modulated for therapeutic benefit.

Perhaps nowhere closer is the reality of clinical translation of basic findings more apparent than in the story of the SUR1regulated TRPM4 ion channel, and its role in the pathogenesis of cerebral edema and hemorrhagic transformation following ischemic stroke (see review by Khanna et al., in this issue). Glibenclamide, an FDA-approved drug commonly used in patients with type 2 diabetes, inhibits SUR1-TRPM4, and several robust preclinical studies have demonstrated the 
efficacy of glibenclamide in reducing edema and hemorrhagic conversion in rodent models of ischemic stroke. This has in turn prompted the study of the potential protective effects of glibenclamide in humans in an ongoing prospective phase II clinical trial, and preliminary data from this effort suggests glibenclamide also significantly reduces ischemic cerebral edema and hemorrhagic conversion [17]. Many other targets listed above are promising candidates at the cusp of human translational studies. We are excited to present this timely resource to the readers of Translational Stroke Research.

\section{Conflict of Interest The authors declare no conflict of interest.}

Open Access This article is distributed under the terms of the Creative Commons Attribution License which permits any use, distribution, and reproduction in any medium, provided the original author(s) and the source are credited.

\section{References}

1. Brian P Walcott MD. Disruption of ion homeostasis in the neurogliovascular unit underlies the pathogenesis of ischemic cerebral edema-TRSR329R. doi:10.1007/s12975-013-0307-9

2. Shan Ping Yu MD PhD. Ionic regulation of cell volume changes and the phenotype of cell death after ischemic stroke-TRSR355R1. doi:10.1007/s12975-013-0314-x

3. Michael Norenberg. Sulfonylurea receptor 1 contributes to the astrocyte swelling and brain edema in acute liver failure-TRSR346. doi:10.1007/s12975-014-0328-z
4. Niyathi Hegde Shah. Voltage-gated potassium channels at the crossroads of neuronal function, ischemic tolerance, and neurodegeneration-TRSR334R1. doi:10.1007/s12975-013-0297-7

5. Zhi-Gang Xiong. Translational strategies for neuroprotection in ischemic stroke-focusing on acid sensing ion channel 1a-TRSR344R1. doi:10.1007/s12975-013-0319-5

6. Xiang-Ping Chu. Acid-sensing ion channels contribute to neurotoxicity-TRSR336R. doi:10.1007/s12975-013-0305-y

7. Nicola J Robertson $\mathrm{MB} \mathrm{ChB}, \mathrm{PhD} . \mathrm{Na}+/ \mathrm{H}+$ exchangers and $\mathrm{pHi}$ in perinatal brain injury: a review-TRSR368. doi:10.1007/s12975-0130322-x

8. Long-Jun Wu. Microglial voltage-gated proton channel Hv1 in ischemic stroke-TRSR323R1. doi:10.1007/s12975-013-0289-7

9. Lucio Annunziato MD. Does $\mathrm{Na}+/ \mathrm{Ca} 2+$ exchanger, NCX, represent a new druggable target in stroke intervention?-TRSR363R. doi:10. 1007/s12975-013-0308-8

10. ShanPing Yu. Ion channels in regulation of neuronal regenerative activities-TRSR364R1. doi:10.1007/s12975-013-0320-Z

11. Keep RF et al. Brain water content. Transl Stroke Res. 2012;3:263-5.

12. Boada FE, Qian Y, Nemoto E, Jovin T, Jungreis C, Jones SC, et al. Sodium MRI and the assessment of irreversible tissue damage during hyper-acute stroke. Transl Stroke Res. 2012;3(2):236-45.

13. Ostrowski RP, Schulte RW, Nie Y, Ling T, Lee T, Manaenko A, et al. Acute splenic irradiation reduces brain injury in the rat focal ischemic stroke model. Transl Stroke Res. 2012;3(4):473-81.

14. Koj H. Inflammation and cerebral aneurysms. Transl Stroke Res. 2013;34:2980-4.

15. Phillips AW, Johnston MV, Fatermi A. The potential for cell based therapy in perinatal brain injury. Transl Stroke Res. 2013;4(2):13748.

16. Jieli Chen MD, Michael Chopp. Angiogenesis and arteriogenesis as stroke targets Transl. Stroke Res. (2012). pp 231-249 Chapter 11.

17. Khanna A, Walcott BP, Kahle KT, Simard JM. Effect of glibenclamide on the prevention of secondary brain injury following ischemic stroke in humans. Neurosurg Focus. 2014;36(1):E11. 